\title{
Valuation of an American Option for the Spanish secondary reserve market using a Machine Learning model
}

\author{
A. Malpica*
}

\begin{abstract}
This paper presents an original methodology to compute a financial product that could enhance the demand side participation in ancillary services, specially for industrial consumers. The financial product consists in an american option on the Spanish secondary reserve market for the following day, where the buyer has the right but not the obligation to offer part of its capacity to the system operator. Considering this approach, an industrial consumer would receive an economic incentive to offer its flexibility to the system without changing its production planning, paying an upfront premium. The computation of the american option is leveraged on a Monte Carlo simulation approach where the random paths are obtained from a machine learning model. The machine learning model attempts to forecast the 24-hour secondary band prices of the following day using a combination of different algorithms; the output of the model is used as a baseline to perform the Monte Carlo simulation that computes the option value.
\end{abstract}

\section{LIST OF SYMBOLS}

$t, \tau \quad$ time - hour of the day

$d \quad$ day

$T \quad$ tenor of the american option, i.e. 24 hours

$i \quad$ index of the random path

$I \quad$ total number of random paths

$g \quad$ index of basis function

$G \quad$ total number of basis functions

$\beta_{r} \quad$ coefficient vector of ridge regression

$\gamma \quad$ shrinkage parameter of ridge penalty

$K \quad$ strike - electricity valuation

$\alpha_{g, t} \quad$ coefficient of basis function $g$ at hour $t$

$\widehat{S}_{t} \quad$ secondary band price prediction at hour $t$

$S_{t} \quad$ secondary band price at hour $t$

$Y_{t} \quad$ error term at hour $t$

$X_{t} \quad$ feature vector at hour $t$

$Z_{t} \quad$ logarithm of the error term at hour $t$

$W_{t} \quad$ input feature vector of the second layer at hour $t$

$V_{t} \quad$ option value at hour $t$

$V_{t, i} \quad$ option value at hour $t$ for random path $i$

$V_{t+\Delta t, i} \quad$ simulated continuation value at hour $t$ for random path $i$

$h_{t} \quad$ payoff of the option at hour $t$

$S_{t, i} \quad$ secondary band price at hour $t$ for random path $i$

$C_{t} \quad$ continuation value at hour $t$

$\widehat{C}_{t, i} \quad$ estimation of the continuation value at hour $t$ for random path $i$

\footnotetext{
*Universidad Pontificia Comillas antoniomalpicamorales@gmail.com - May, 22, 2016
}

$\widehat{C}_{0} \quad$ option value estimation

$b_{g} \quad$ basis function of grade $g$

$M A E_{d} \quad$ mean absolute error of day $d$

\section{INTRODUCTION}

$\mathbf{T}$ raditionally, in electric power systems, demand side has been regarded as a passive agent without any obligation to contribute to system reliability and operation. Generators were responsible for supplying the demand, the security of supply and the balancing services to cover demand fluctuations, intermittent generation and network outages.

According to [1], demand side management (DSM) is aimed to adapt energy consumption to improve overall electricity usage and infrastructure efficiency through the implementation of policies and methods that control electricity load. DSM could be driven by energy efficiency and demand response (DR) programmes; whilst the former refers to decrease the amount of energy consumed to end products or services, the latter attempts to shift in time end consumption in response of external signals [2].

DR can be further broken down into price based and incentive based programmes. In the former, a consumer is responsible to manage its own consumption with regard to a pricing mechanism that fluctuates according to real time cost of electricity. On the other hand, incentive based programmes are arranged to provide ancillary services or maintain the security of supply in emergency or contingency situations, through an established market or via bilateral contracts. In these markets or contracts, the consumers provide the service, but the load management is executed by a third party, e.g. the transmission system operator (TSO) or an aggregator.

Notwithstanding, a detailed knowledge of potential customers is required in order to implement successful DR programmes. Therefore, technical studies regarding the end use of electricity should be carried out to characterise the demand and identify the flexibility and the suitable DR method to apply. This emphasises the complexity in the demand participation in electricity markets [3]. Due to this complexity, the mainstream approach of DR research and application has been to address small residential loads through aggregation to achieve a significant volume that can impact on system operation [4].

Industrial consumers are a promising target who can benefit from DR programmes, due to the vast uptake of electricity. As mentioned in [5], two criteria identify large industries with 
economic potential for DR: the total electricity demand and the specific costs for energy. Consequently, electricity cost is essential for these industries and they can obtain economic benefits offering part of their flexibility in the electricity markets or adapting their consumption profile according to external signals. Within industrial consumers, there is a cluster known as electro-intensive consumers; these industries perform processes such as chemical production, steel and non-ferrous metal manufacturing that require an intensive use of electricity.

In [6], real examples of large industries performing DR actions are highlighted. Several articles [7-12] aim to model the flexibility contribution together with the computation of possible economic benefits and the feasibility of the DR programmes. All these studies stress the difficulty on assessing the internal flexibility of industrial consumers, because a detailed knowledge of the industrial process is required; in addition, each electro-intensive industry must be analysed at a time, and the effective DR programmes to be applied should be adapted specifically. Hence, the feasibility of each DR programme in the literature review is conditioned to a tailored solution for the consumer.

Therefore, the objective of this paper is to propose a general framework for a DR programme that could be easily applied for any industry. Due to the difficulty to assess the internal flexibility of industrial consumers, the proposed model resorts to an external market vision. In this case, the proposed solution consists in a financial option where the underlying asset is the secondary band price of the Spanish electricity system, reflecting the participation of the industrial demand in this ancillary service. At the same time, the possibility of exercising or not the option provides flexibility in the operation for the industrial consumer. This paper is structured as follow: Section II introduces the description of the financial product. The methodology is presented in section III, where the mathematical formulation and the machine learning model used are described. Results of a case study are shown in section IV. Finally, section V presents the conclusions of the study.

\section{FINANCIAL PRODUCT DESCRIPTION}

Typically, financial products are traded within electricity systems between generation and demand agents who want to hedge against uncertainty and volatility. A literature review of derivatives in electricity markets [13-16] reveals that, large consumers who need a fixed price for electricity can resort to future, forward contracts or options to remove really high peak prices that could reduce their operating margins; on the other hand, retailers or aggregators can settle options to mitigate consumers load deviations or significant forecasting errors that yield economic losses.

The financial contract described here is settled on the secondary reserve market between the Spanish TSO and a large industrial consumer who is willing to participate in this market. The secondary regulation is an ancillary service that aims to guarantee the equilibrium between generation and demand, and handles the deviations from real-time operation. The secondary regulation consists on two products: up and

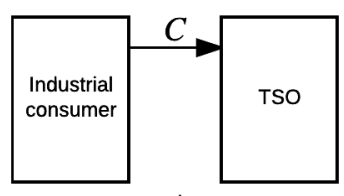

a)

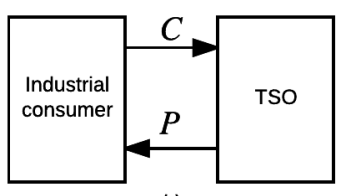

b)
Fig. 1: Payments between the TSO and an industrial consumer.

down secondary band (capacity) and up and down secondary energy (use). The focus of this study lies on the secondary band while the secondary energy use is out of scope. The secondary band is procured by the TSO on a daily basis; for each hour of the day, the TSO defines different levels of required band. The mechanism to determine which agents will provide the secondary band is a market held one day before delivery that is cleared marginally.

Though demand side is not currently allowed to participate in this ancillary service in Spain, there are plans for a future opening to these kind of markets, coming from EU policies and directives [17], together with good experiences in other countries. Then, the scenario suggested here proposes a framework where industrial consumers can participate on these ancillary services. The financial product, that could enable demand side in general and large industrial consumers in particular to contribute to system operation, consists of an american option on this day-ahead secondary reserve market. As a result, an industrial consumer can buy an american option to offer part of its load on one hour of the secondary band market. The american option gives the industrial consumer the right but not the obligation to exercise the option at any time up to the expiration date [18]. In this case, the industrial consumer can allocate part of its demand on the secondary band upon request of the TSO, at any time on the 24 hours of the following day. Since the industry does not have the obligation to exercise the option, inherently it acquires flexibility to manage its production planning without being subject to possible load shedding from the secondary band deployment.

Two outcomes might result after the option arrangement, with different payoffs for the counterparties. Figure 1 represents the payments direction of the two scenarios. In both scenarios, the industrial consumer pays an upfront premium to have the aforementioned right; this upfront premium is the option value $C$ that will be describe and compute later. This payment prevents from a risk-free profit for the industrial consumer. In Figure 1 a), the industrial consumer ends up not exercising the option, thus not receiving any payment from the TSO; this situation might happen when the industrial manufacturing is prioritised rather than the possible revenue coming from secondary band participation or the industrial agent does not find economically attractive the hourly prices of the secondary band after the publication thereof. On the other hand, in Figure $1 \mathrm{~b}$ ), the industry decides to exercise the option because it finds more profitable allocating part of its load on the secondary band and being exposed itself to possible load reduction. As a result, if the industrial consumer is capable to maintain the arranged load, the TSO will pay 
the industrial consumer $P$, which is the hourly price of the secondary band times the amount offered by the industrial consumer.

\section{Methodology}

This section introduces the methodology followed to compute the aforementioned option value $C$. Since the hourly secondary band price is the underlying of the american option, the first step is to obtain a mathematical expression that represents its behaviour. This behaviour will be driven by a stochastic process that will help to further evaluate the option through a Monte Carlo simulation.

\section{A. Mathematical formulation}

This section will obtain a expression for the hourly secondary band price $S_{t}$. Most state of the art focus on spot prices, as this is the basic and most common electricity product, underpinned by the fact that spot prices exhibits characteristics such as seasonal pattern, mean-reversion effect and price spikes [19]. The stochastic process of the spot price $P_{t}$ is often represented as (1), where $f(t)$ is a known deterministic function of time and $Q_{t}$ is a diffusion stochastic process such as a standard Brownian motion [20]. Modifications of this process can also be found in [21] and [22], where the first study incorporates a mean-reverting with jumps that represents the price spikes and the second study adds an autoregressive model for the logarithm of the energy price.

$$
P_{t}=f(t)+Q_{t}
$$

However, these models attempt to obtain an exact mathematical equation that describes the spot electricity prices, relying on the predictable component with the implication it has for derivative valuation. In [20] the predictable component is described exhaustively; the deterministic part is fitted as a linear regression to try to capture the seasonalities of the process as much as possible. In addition, the stochastic process is discretised following an autoregressive model of order 1.

Three issues might arise if this kind of approach is followed to obtain the secondary band price model. First of all, these model have been derived from spot electricity prices, and in this study, the price to forecast is the secondary band, whose market is governed by different mechanisms and the behaviour of the agents might change. Then, the deterministic component could be very biased, since it just tries to identify the seasonal pattern through a linear regression looking at the kind of day and the calendar month, leaving aside possible electricity system variables that could enhance the prediction. Finally, considering that the electricity prices for the secondary band are obtained via an auction for the 24 hours of the following day; this modeling could not be applied as there would be a lack of information, e.g. in order to predict the price for the hour seven of the following day, the price of the hour six must be available and all prices for the following 24 hours are cleared simultaneously.

The proposed model relies on a Machine Learning (ML) algorithm that predicts the values of the hourly prices of the secondary band for the following day. The ML algorithm structure and the used data are described in detail in the following subsection. The ML model is trained to forecast $S_{t}$, for the 24 hours of the following day.

As the outcome of the model is a single value for each hour, the stochastic process disappears using this technique; notwithstanding, the ML approach has an inherent error that can be used to restore the stochastic process that will be required to perform a Monte Carlo simulation.

Let $S_{t}$ be the hourly secondary band price and $\widehat{S}_{t}$ the outcome of the model, the error of the model can be represented as (2), where $Y_{t}$ is the error term. A logarithmic transformation is applied to both sides of the equation. (3) shows that the output of the ML model is a function of $X_{t}$, being $X_{t}$ the vector of features that enters into the model; subsequently, the ML model finds a suitable function, $f(\cdot)$, that maps the input data to the output value, while minimising the error between the prediction $\widehat{S}_{t}$ and the true value $S_{t}$. Moreover, changing $\operatorname{Ln}\left(Y_{t}\right)$ for $Z_{t}$, rearranging (2) and combining with (3), the desired mathematical expression for $S_{t}$ is represented in (4).

$$
\begin{gathered}
\frac{S_{t}}{\widehat{S}_{t}}=Y_{t} \\
\operatorname{Ln}\left(\frac{S_{t}}{\widehat{S}_{t}}\right)=\operatorname{Ln}\left(Y_{t}\right) \\
\widehat{S}_{t}=f\left(X_{t}\right) \\
S_{t}=f\left(X_{t}\right) \cdot e^{Z_{t}}
\end{gathered}
$$

This equation may be quite similar to that presented in (1), however, here the deterministic part is obtained through a ML model that has no so easy interpretation as detailed in [20]. Furthermore, the hypotheses behind the ML approach assume that the errors in the model are independent and identically distributed from a normal random variable with mean zero and variance $\sigma^{2}$.

\section{B. Machine learning algorithm}

Machine learning can be defined as a set of methods that can automatically detect patterns in data, and use the covered patterns to predict future data or perform other kind of decision making, such as classification. The type of ML model used in this study corresponds to a supervised learning, where the underlying statistical model uses a set of inputs with its related outputs as examples to learn patterns in data that allow it to predict accurately the output of future examples, for which their results are not known [23].

Typically, in regression problems, an objective function to minimise the difference between the true output of the data and the predicted output from the model is used in order to ensure the model adjusts well to the data. The group of data used to learn the patterns is called the training set, and the result of the cost function is known as training error. However, an undesired effect might arise if the model learns too much, meaning that the model adjusts excessively to the known output of the data that it does not generalise well when future data are predicting, 


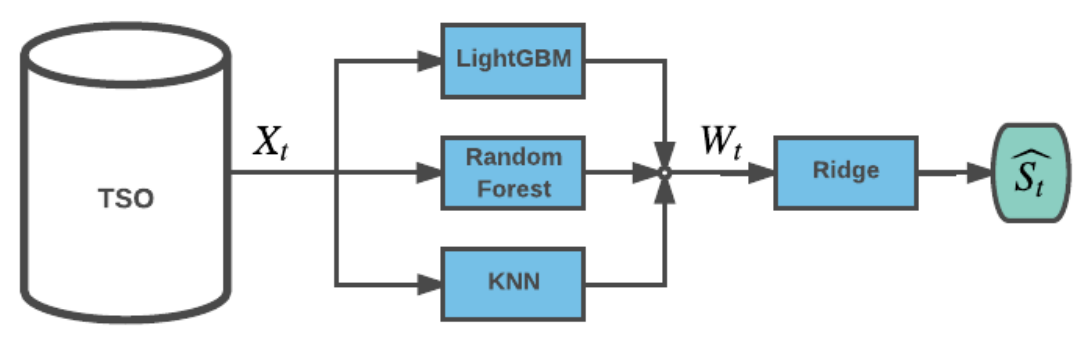

Fig. 2: Structure of the Machine Learning model for the Secondary band prediction $\widehat{S}_{t}$.

yielding a very poor performance or test error, which is the result of the cost function applied to new observations that the model has never seen; this effect is known as overfitting.

In order to avoid overfitting, a cross-validation (CV) technique must be carried out to ensure the model generalises properly. CV is a mathematical method that can estimate the test error by removing a group of samples from the training set, train the model on the remaining set and predict on the hold out set. There are different CV techniques that can be applied, however, given the characteristics of the problem, i.e. it can be seen as a time series problem, the $\mathrm{CV}$ technique consists in training the model up to a certain day, then predicting on the following day, checking the model performance on the test and repeating this process adding the samples of the following day. Hence, the CV can be seen as training on a rolling window that extends up to each new day, as new examples come from the secondary band market.

Figure 2 represents the ML structure. The input data comes from historical records of the Spanish TSO [24]. Three different ML algorithms (a LGBM, a random forest and a k-nearest neighbours) are trained with the data, and each outcome thereof is finally combined with meta-data (hour of the day, and day of the week) and used to trained a linear regression with ridge regularisation. This final layer yields the hourly secondary band price prediction. Below, a brief summary of the input data, the ML algorithm and the american option valuation process are highlighted for a better understanding of the model.

1) Input data: Since, this study has an objective to forecast the secondary band price for the following day, real data from electricity Spanish system are obtained on a hourly basis. Notwithstanding, the model can be always update up to a new date due to each day, new observations can be incorporated into the model. A combination of raw hourly observations and some feature engineering (values of the previous hour, values of the same hour for the day before, moving averages) is carried out to define the vector $X_{t}$ of features; a detailed list of all inputs can be found in the Appendix. The length of the vector $X_{t}$ is 85 , meaning that the algorithms have to deal with 85 different inputs variables for each observation.

2) ML algorithms: The collected data are preprocessed and fed in the three aforementioned ML algorithms, that composed the first layer of the model. These algorithms receive the same input, however each one deals with the data in a different way, producing an estimation of the hourly secondary band price.
These three models [25-27] are standard in the industry, and all packages used are open source.

Due to the distinct behaviour of each algorithm, the performance of every model might produce different predictions regarding the secondary band price. A direct aftermath of using different models in the same data set is that one model can perform well under certain conditions, yielding a good prediction for some observation and for the same conditions, another model can perform worse, diverting from the true output reasonably and vice versa. Thus, combining the outputs of all models into another model might increase the overall performance; this technique is known as stacking [28].

A linear regression is used as the model in the final layer. The ridge name refers to an additional penalty that is included to the objective function, as shown in (5), where $W_{t}$ is the input feature from the first layer, $\gamma$ is a non-negative parameter that reduces the weight of the coefficient and $\|\cdot\|_{2}$ is the euclidean norm. Another interesting effect, is that the coefficient vector $\beta_{r}$ does not depend on time, i.e. the model is trained to yield a coefficient vector that generalises regardless of the hour to predict, giving a robust model.

$$
\min _{\beta_{r}}\left\|S_{t}-W_{t} \beta_{r}\right\|_{2}^{2}+\gamma\left\|\beta_{r}\right\|_{2}
$$

\section{American option value}

Now, the ML model is able to generate the desired prediction of the hourly secondary band price for the following day, $\widehat{S_{t}}$. In (4), a stochastic process takes place through $Z_{t}$; thus, a Monte Carlo simulation allows to valuate the american option for the industrial consumer. The value of an option is the discounted expected payoff under the risk-neutral measure; for simplicity, no risk-free rate has been assumed, resulting the option value in just the expected payoff.

The value of the american option can be stated as (6), where $V_{0}$ is the option value at time zero, $\mathbf{E}_{\mathbf{0}}(\cdot)$ is the expected value, $S_{\tau}$ is the secondary band price at time $\tau$, and $h_{\tau}$ is the payoff of the option at time $\tau$, that is represented in (7), being $K$, the strike value. $T$ is the total time that the window of the american option is opened, i.e. 24 hours in this study, and $\tau$ is each one of the possible hours in which the option can be exercised.

$$
V_{0}=\sup _{\tau \in\{0, \Delta t, 2 \Delta t, \ldots, T\}} \mathbf{E}_{0}\left(h_{\tau}\left(S_{\tau}\right)\right)
$$




$$
h_{t}\left(S_{t}\right)=\max \left(S_{t}-K, 0\right)
$$

The option value problem becomes an optimal stopping problem. The algorithm used to solve this problem is called Least-Squares Monte Carlo [29]. It can be shown that the american option value at any point in time is the maximum between the payoff in that moment and the continuation value (8), being the index level of the secondary band price $s$. At any possible exercise time, the buyer of the american option can compare the immediate exercise of the option, with the expected payoff for not exercising the option now, and continuing to keep the option alive. This conditional expectation (9) can be estimated from the cross-sectional information that the simulation provides.

$$
\begin{gathered}
V_{t}(s)=\max \left(h_{t}(s), C_{t}(s)\right) \\
C_{t}(s)=\mathbf{E}_{t}\left(V_{t+\Delta t}\left(S_{t+\Delta t}\right) \mid S_{t}=s\right)
\end{gathered}
$$

Assuming that the simulation has $I$ paths of the underlying over $T$ time intervals of equal size $\Delta t . V_{t+\Delta t, i}$ is the simulated continuation value for path $i$ at time $t$; however, this number cannot be used due to the perfect valuation foresight it would mean. Instead of this number, the cross-section of all simulated continuation values can be used to approximate the expected continuation value by least-squares regression.

Consequently, the least-squares optimisation can be stated as (10), where the continuation value is estimated as $\widehat{C}_{t, i}=$ $\sum_{d=1}^{D} \alpha_{g, t}^{*} b_{g}\left(S_{t, i}\right)$, being $b_{g}$ a set of basis function (e.g., $\mathrm{g}=2$ implies the square value of $\left.S_{t, i}\right)$ and $\alpha_{g, t}^{*}$, the solution obtained from the minimisation problem.

$$
\min _{\alpha_{1, t}, \ldots \alpha_{G, t}} \frac{1}{I} \sum_{i=1}^{I}\left(V_{t+\Delta t, i}-\sum_{g=1}^{G} \alpha_{g, t} b_{g}\left(S_{t, i}\right)\right)^{2} \quad \forall t
$$

Generally speaking, the Least-Square Monte Carlo approach can be synthesised in the following steps:

1) The $I$ random paths are computed for the 24 period.

2) Starting from the last period, the expected payoff of immediate exercise and the estimated continuation value are compared and kept the maximum of both.

3) This process is repeated backwards, and for each hour, the continuation value $\widehat{C}_{t, i}$ is estimated using the cross-section information.

4) The option value can be calculated averaging all random paths, that is, using the Monte Carlo estimator, as shown in (11).

$$
\widehat{C}_{0}=\frac{1}{I} \sum_{i=1}^{I} V_{0, i}
$$

This option value $\widehat{C}_{0}$, is the premium that the industrial consumer will be willing to pay upfront in order to hold the american option. It has to be noted that the option value is strongly dependent of the secondary band price (in this case the estimation resulted from the ML model) and the strike $K$. Each industrial consumer has an intrinsic electricity value that depends on the industrial process performed. However, this intrinsic value relies on several factor that may be difficult to take into account. As a result, every industrial consumer will have a different electricity valuation, represented here as the strike $K$, as the strike might stand for the payment that the industrial consumer will have to receive in order to reduce one unit of electricity energy.

\section{CASE STUDY}

In this section, the case study is presented together with the results obtained. First a brief explanation of the case study considered is introduced. Then, the results are explained. Finally, several examples of the american option valuation are shown.

\section{A. Case study description}

As it has mentioned before, the case study is carried out for the Spanish electricity system, particularly for the deployment of an american option based on the secondary reserve market, where the capacity band is cleared and assigned on a daily basis, resulting in a price signal in EUR/MW for each one of the hour of the following day.

A list of the main input variables used in this study can be seen in the Appendix. The length of the observed period is from January 1, 2016 until April 29, 2017. As the model is trained to predict on a daily basis, the input data is fed until the desired day to predict. The first predicted date corresponds to January 31, 2016; so, for the first prediction a set of 30 days is hold as training set. As time goes by, new input dates can be incorporated into the model, hence, for April 29, 2017 all previous dates are fed into the model as examples to train. With the forecasts obtained from the ML model, the american option can be computed, meaning that for each day an american option can be evaluated.

\section{B. ML model accuracy}

The ML model must be validated in order to confirm that the model works properly and it yields a good approximation of the secondary band price. As it has been mentioned before, the $\mathrm{CV}$ strategy followed lets to check for each day which is the precision of the model. The model performance is evaluated through the mean absolute error (MAE) on a daily basis, as shown in (12). For each day $d$, the model predicts without knowing the true output of the secondary band price; once the hourly secondary band price are published, the performance can be tested through the error achieved.

$$
M A E_{d}=\frac{1}{24} \sum_{t=1}^{24}\left|S_{t}-\widehat{S}_{t}\right| \quad \forall d
$$

Considering that each one of the proposed algorithms looks at the same target (secondary band price), the previous error assessment can be done for the four algorithms; this also highlights the fact that the stacking procedure yields a lower error rate, turning into a higher accuracy. Figure 3 shows the daily MAE obtained for all days predicted by the model. It can be seen that the MAE decreases as the entire model has more data to learn, as the new daily observation are added 


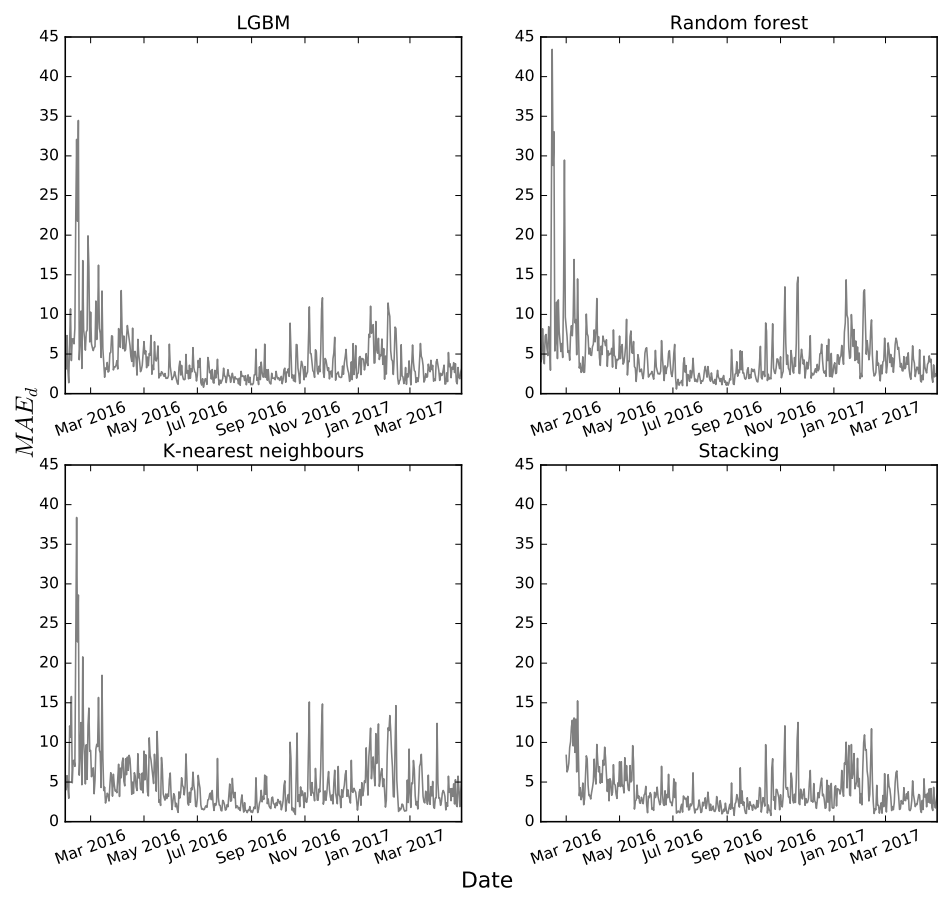

Fig. 3: Daily mean absolute error obtained from the different algorithms.

to the training set. In addition, the model with the lower average rate is the stacking, followed by the LGBM, showing less oscillations, thanks to the contribution of the models on the previous layer. The almost negligible difference between the LGBM and the stacking reflects that this is the model leveraging the bulk of the stacking model.

Regarding that the main objective of the ML model is to predict the hourly prices of the secondary band market, then it is mandatory to check how well the ML model output seems to the market prices. For this purpose, Figure 6 shows the real market prices of the secondary band, together with the daily out-of-sample predictions that the ML model generates. It can be seen that the predictions resemble fairly to the secondary band pattern, i.e. the ML model understands the hidden patterns of the band and is able to produce a prediction with some confidence interval around the true secondary band price.

Obviously, the predictions are not $100 \%$ accurate and they would not match exactly the secondary band even with the best possible model, as the statistical modelling assumes always an irreducible error that cannot be removed. Having a similar pattern of the secondary band market is enough for this study, because of the later Monte Carlo simulation approach will generate a lot of random paths obtaining an expected value from them. Henceforth, the true secondary band price will be embedded among all random paths.

\section{Option valuation}

Once the accuracy of the ML model has been demonstrated, the american option valuation has to be computed. The length of the simulation is fixed to 24 hours, as the american option can be exercised during the whole following day. Since a very large number of random paths increases the accuracy of the option value estimation at the expense of higher computational requirements, 100,000 independents random paths have been selected. Finally, 3 are the number of basis functions used to estimate the continuation value of (10).

Figure 4 - 5 represent the Monte Carlo simulation performed, together with the option value obtained for two different days. On the upper graph, the blue line represents the output from the ML model, while the green line is the real secondary band prices resulting from the market; the gray lines are just a $10 \%$ of all random paths computed. On the graph below, the option value is calculated for different strike levels using the aforementioned Least-Squares Monte Carlo algorithm; therefore the curve represents the option value, i.e. the upfront premium that the industrial consumer will have to pay in order to hold the american option, for different strikes level, where, again the strike level can be viewed as the intrinsic electricity value.

On average, the estimation of the secondary band price from the ML is close to the true output, resulting in a good approximation for the expectation of the random paths, leading to a reliable american option valuation. Regarding the option value - strike curve of the different american options evaluated, it can be seen that both exhibit a similar behaviour. For very large strikes prices (electricity valuation) the american option value is zero; as the strike starts to reduce, the american option value tends to increase until reaching almost a straight line trend, crossing an elbow area. This elbow area usually starts around the highest value of the prediction from the ML model, however the shape of the entire curve depends on the generated random paths.

These option value - strike curves can be used as a quick visual tool to determine if it results valuable to enter into the financial contract of the american option; for a given strike, the premium is almost immediate to obtain and vice versa. The curves are computed with forecasts that are underpinned by real market data, thus, all insights that could be drawn rely on market side information instead of the limited and not scalable information that could be derived from an industrial load study, that could not be replicated easily.

Considering the utilisation by the industrial consumers, which are the group of interest in this study, it makes sense, that for those industrial consumers with a very high electricity valuation (essential implication of electricity in their process and intensive costs too) the upfront payment of the option would be zero, since just participating in this market with the cleared prices yields an economic loss for this group. Notwithstanding, those industrial consumers with a large utilisation of electricity but a cheaper electricity valuation, have an opportunity to exercise the american option and receive the secondary band market price, resulting in a possible profitable situation; though the profitability will depend on further events that are out of scope of this study.

Also, this option value-strike curve can be interpreted as the upfront payment that the industrial consumer would have to pay in order to be hedged and end in a zero profit situation. If an industrial consumer had an electricity valuation of $K$, assuming that the industrial consumer would be curtailed 1 

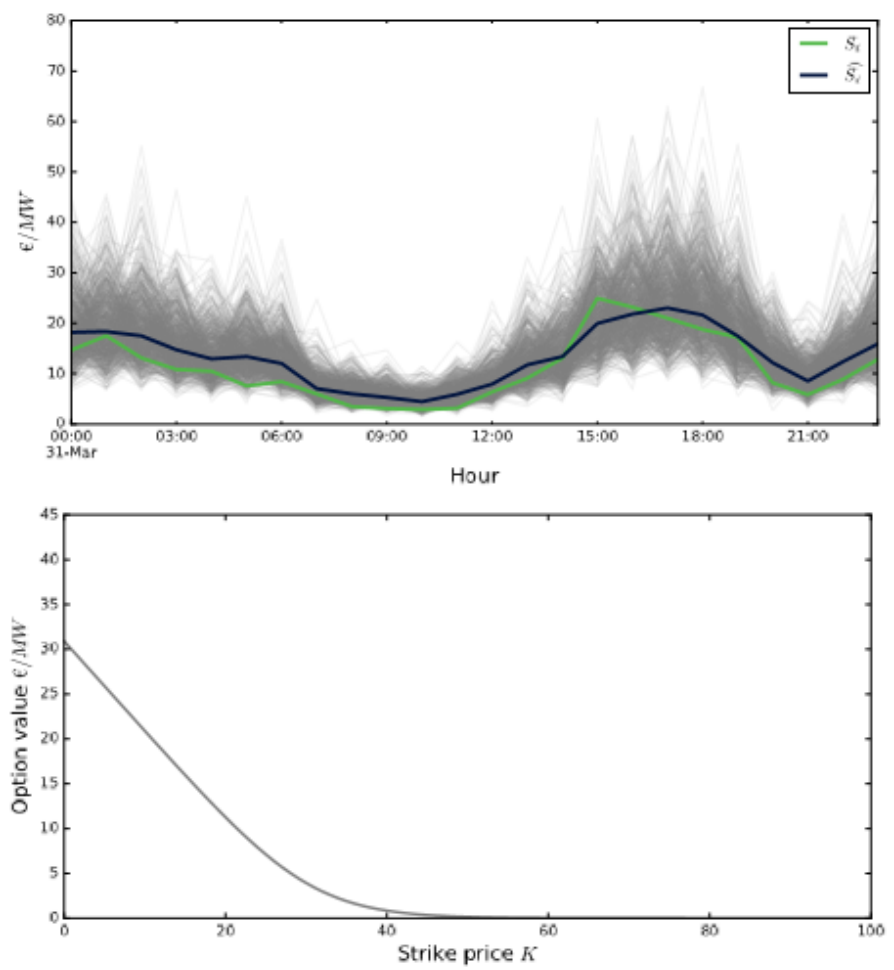

Fig. 4: Monte Carlo simulation and Option value against strike 2017-03-31

MW of electricity during one hour, this $K$ would represent the amount lost by the consumer, therefore it would be willing to receive at least this amount $K$ to participating in the secondary reserve market to end without economic losses in case of the TSO would deploy its load. Regarding the curves and also the output from the ML model, it can be seen that the option value is almost zero for strikes values higher than the largest value of the ML output. This seems reasonable as for these high strike values, the industrial consumer will be unable to recover the possible cost due to a load reduction performed by the TSO. In addition, the option value is not zero at the peak value of the estimation, and it always presents an extra charge that would avoid to the industrial consumer to turn out in a risk-free profit situation.

\section{CONClusion}

An innovative methodology to value an american option for the Spanish secondary band market has been proposed in this study. After carrying out the required calculations to end with a solution and analysing it, several conclusions can be drawn, which are stated now.

The new approach proposed here lies on an existing market point of view. Instead of developing a tailored solution that accounts for the flexibility of the consumer, the stated solution consists in a general framework where the flexibility of the industrial consumer is considered and can be integrated in the system operation. For that, each large industrial consumer will have to perform an internal audit of its processes to find where they have this flexibility and if it is feasible to deploy it on a daily basis or coinciding with a maintenance stop.
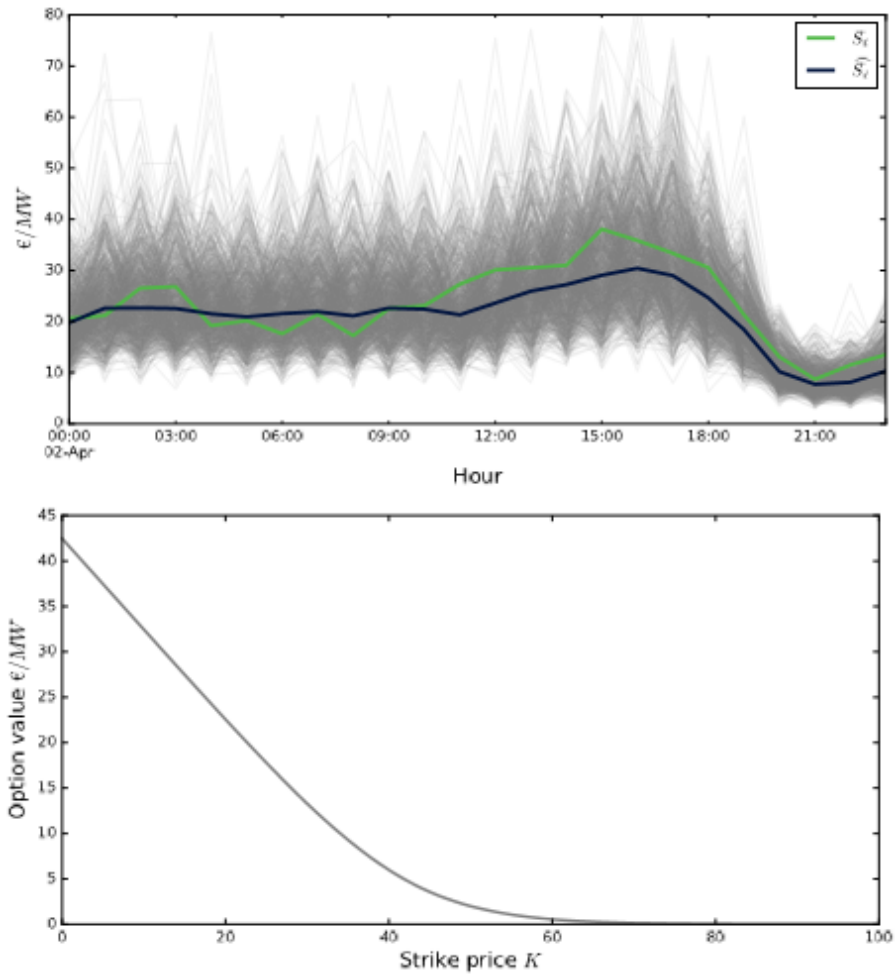

Fig. 5: Monte Carlo simulation and Option value against strike 2017-04-02

The advantage lies on that any industrial consumer can know quickly the upfront premium, based on its electricity valuation, that would have to pay in order to hold the american option, and accordingly to this price decide whether to settle the financial contract, enabling the TSO to consider a possible load reduction coming from industrial consumers.

Consequently, the option value-strike curves shown in Figures 4 - 5 provide information and insights for both industrial consumers and the TSO. Industrial consumers can obtain the lower bound of the price they would be willing to offer based on their electricity value, as the option value represented can be seen as a benchmark. On the other hand, the TSO can anticipate the revenues it could receive via selling the american options to the industrial consumers.

The american option valuation is underpinned by a ML model whose output is the forecasting of the secondary reserve market prices for the following day. It has been proven that the model predicts the secondary band market pattern similar to the true market prices, however the model does not predict exactly the same prices due to the irreducible error of the underlying statistical model. The ML model is robust, as the mean absolute error accumulated since the first predictions until the last validation day is $3.813 \mathrm{EUR} / \mathrm{MW}$, meaning that on average the model tends to over or underestimate the price in that quantity. Another advantage of the ML model is that it performs better as more observations and samples are included in the pipeline to predict, i.e. more days.

Finally, the american option valuation shows that the resulting option value is such the industrial consumer could end in a zero profit situation, as it was used as a hedge 


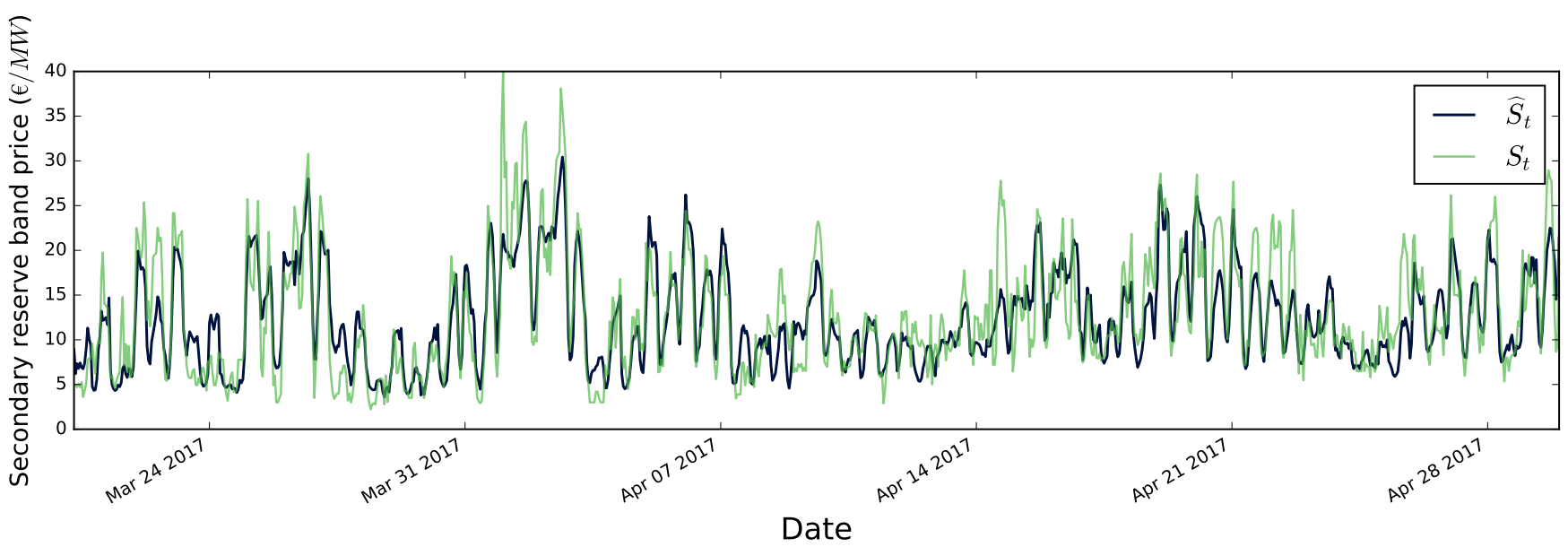

Fig. 6: Secondary band predictions $\widehat{S}_{t}$ and real secondary band market prices $S_{t}$.

instrument. However, more analysis must be carried out to take into account the potential benefits for the counterparties; those analysis should include the possible deployment of the industrial load by the TSO, assessing the profit and loss for the industrial consumer with the subsequent payments that the TSO should make; also a cost and benefit analysis for all agents involved in the system if this kind of financial product is incorporated to the system operation.

Beforehand, it seems that keeping both the industrial demand and traditional generators in secondary reserve might result in a cost overrun for the system; however the analysis of different scenarios should underpinned the deployment of this solution or not. For example, if an industrial consumer has paid the option, but it does not exercise it, this supposes an earning for the TSO that could reduce system cost; another situation would be prioritising the use of the demand from industrial consumers participating in this service, as it would avoid increasing generation from conventional power plants, with the environmental benefits it brings together with the savings from replacing tertiary reserves. In the end, further analysis and improvements must be carried out thoroughly, because this framework can be replicated and applied for other agents that could be interested in this product, such as aggregators or even generators.

\section{APPENDIX}

Here, the variables used to define $X_{t}$ for the ML model are presented. Table I contains a list of the raw variables obtained from the Spanish TSO; in addition, a feature engineering process has been performed and for each hourly sample observation, the following variables are included when it is possible as inputs. PVP stands for daily provisional feasible programme.

1) The same variables of the previous hour (-1 hour).

2) The same variables of the previous day at the same hour (-24 hour).

3) The moving average value of these variables during the last 24 hours.
TABLE I: Base input variables for the ML model

\begin{tabular}{|c|c|}
\hline Variable & Description \\
\hline band & Secondary band price \\
\hline spot & $\begin{array}{c}\text { Spot price for the following day (Day-ahead } \\
\text { market) }\end{array}$ \\
\hline coal & Coal generation from PVP \\
\hline ccgt & $\begin{array}{l}\text { Combined cycle gas turbine generation from } \\
\text { PVP }\end{array}$ \\
\hline wind & Wind generation from PVP \\
\hline nuclear & Nuclear generation from PVP \\
\hline solar & Solar generation from PVP \\
\hline hidraulic & Hidraulic generation from PVP \\
\hline installed_hidraulic & Hidraulic installed capacity \\
\hline installed_coal & Coal installed capacity \\
\hline installed_ccgt & $\begin{array}{l}\text { Combined cycle gas turbine installed } \\
\text { capacity }\end{array}$ \\
\hline demand & Programmed demand from PVP \\
\hline programmed_demand & $\begin{array}{l}\text { Programmed demand forecasted by the TSO } \\
\text { one day in advance }\end{array}$ \\
\hline spot_energy & Energy cleared in the day-ahead market \\
\hline forecasted_wind & Forecasted wind production by the TSO \\
\hline forecasted_solar & Forecasted solar production by the TSO \\
\hline france & $\begin{array}{l}\text { Net exchange with French interconnection } \\
\text { from PVP }\end{array}$ \\
\hline portugal & $\begin{array}{l}\text { Net exchange with Portuguese } \\
\text { interconnection from PVP }\end{array}$ \\
\hline morocco & $\begin{array}{l}\text { Net exchange with Moroccan } \\
\text { interconnection from PVP }\end{array}$ \\
\hline
\end{tabular}

\section{REFERENCES}

[1] Eurelectric, "Eureletric views on demand-side participation," 2011.

[2] C. Battle and P. Rodilla, "Electricity demand response tools: Status quo and outstanding issues," European Review of Energy Markets. Special Issue on Incentives for low-carbon energy future, 2008.

[3] S. Valero, M. Ortiz, C. Senabre, C. Alvarez, F. Franco, and A. Gabaldón, "Methods for customer and demand response policies selection in new electricity markets," IET Generation, Transmission \& Distribution, vol. 1, no. 1, pp. 104-110, 2007.

[4] Alagna, V. et al., "Addess EU Project. Description of market mechanism which enable active demand participation in the power system. D5.1," 2011.

[5] M. Paulus and F. Borggrefe, "The potential of demand-side management in energy-intensive indsutries for electricity markets in germany," Applied Energy, vol. 88, pp. 432-441, 2011.

[6] T. Samad and S. Kiliccote, "Smart grid technologies and applications for the indsutrial sector," Computers and Chemical Engineering, vol. 47, pp. 76-84, 2012. 
[7] S. Mohagheghi and N. Raji, "Dynamic demand response solution for industrial customers," in Industry Applications Society Annual Meeting, Lake Buena Vista, 2013, pp. 1-9.

[8] M. Choobineh and S. Mohagheghi, "Optimal energy management in an industrial plant using on-site generation and demand scheduling," IEEE Transactions on Industry Applications, vol. 52, no. 3, pp. 1945-1952, 2016.

[9] X. Zhang, G. Hug, Z. Kolter, and I. Harjunkoski, "Industrial demand response by steel plants with spinning reserve provision," in North American Power Symposium (NAPS), Charlotte, NC, 2015, pp. 1-6.

[10] S. Ashok, "Peak-load management in steel plants," Applied Energy, vol. 83, no. 5, pp. 413-424, 2006.

[11] F. Yuan Xu and L. Lei Lai, "Novel active time-based demand response for industrial consumers in smart grid," IEEE Transactions on Industrial Informatics, vol. 11, no. 6, pp. 1564-1573, 2015.

[12] D. Logenthiran, D. Srinivasan, and T. Zong Shun, "Demand side management in smart grid using heuristic optimization," IEEE Transactions on Smart Grid, vol. 3, no. 3, pp. 1244-1252, 2012.

[13] T. Chung, S. Zhang, C. Yu, and K. Wong, "Electricity market risk management using forward contracts with bilateral options," IEE Procedings - Generation, Transmission and Distribution, vol. 150, no. 5 , pp. 588-594, 2003.

[14] S. Pineda and A. Conejo, "Using electricity options to hedge against financial risks of power producers," Journal of Modern Power Systems and Clean Energy, vol. 1, no. 2, pp. 101-109, 2013.

[15] C. Olofsson, "Pricing swing options in the electricity market," Master's thesis, Lund University, Lund, Sweden, 2015.

[16] S. Oren, "Integrating real and financial options in demand-side electricity contracts," Decision Support Systems, vol. 30, pp. 279-288, 2011.

[17] J. Torriti, M. Hassan, and M. Leach, "Demand response experience in Europe: Policies, programmes and implementation," Energy, no. 35, pp. $1575-1583,2010$

[18] J. Hull, Options, futures and other derivatives. Upper Saddle River, NJ, USA: Pearson/Prentice Hall, 2009.

[19] U. Dörr, "Valuation of swing options and examination of exercise strategies by monte carlo techniques," Master's thesis, Univeristy of Oxford, Oxford, 2003.

[20] J. J. Lucia and E. S. Schwartz, "Electricity prices and power derivatives: Evidence from the Nordic power exchange," Review Deriv. Research, no. 5, pp. 5-50, 2002.

[21] B. Hambly, S. Howison, and T. Kluge, "Modelling spikes and pricing swing options in electricity markets," Quantitative Finance, vol. 9, no. 8, pp. 937-949, 2009 .

[22] N. Meinshausen and B. Hambly, "Monte Carlo Methods for the Valuation of Multiple Exercise Options," Mathematical Finance, no. 14, pp. 557-583, 2004.

[23] K. P. Murphy, Machine Learning: A probabilistic Perspective. Cambridge, Massachusetts, USA: The MIT Press, 2012.

[24] Red Eléctrica de España. (2017) Esios. Sistema de información del Operador del Sistema. [Online]. Available: https://www.esios.ree.es/es

[25] Microsoft. (2017) Microsoft / LightGBM. [Online]. Available: https://github.com/Microsoft/LightGBM

[26] Scikit learn. (2016) Scikit learn - 1.11. Ensemble methods. [Online]. Available: http://scikit-learn.org/stable/modules/ensemble.html

[27] — . (2016) Scikit learn - 1.6. Nearest Neighbors. [Online]. Available: http://scikit-learn.org/stable/modules/neighbors.html

[28] J. Sill, G. Takacs, L. Mackey, and D. Lin, "Feature-weighted linear stacking," 2009. [Online]. Available: https://arxiv.org/pdf/0911.0460.pdf

[29] F. Longstaff and E. Schwartz, "Valuing american options by simulation: A simple least-squares approach," Review of Financial Studies, vol. 14, no. 1, pp. 113-147, 2001. 